\title{
Erratum
}

\section{Remark on the Absence of Absolutely Continuous Spectrum for $d$-Dimensional Schrödinger Operators with Random Potential for Large Disorder or Low Energy}

F. Martinelli ${ }^{1}$ and E. Scoppola ${ }^{2}$

Laboratoire de Physique Théorique et Hautes Energies ${ }^{3}$, Université Pierre et Marie Curie, 4 Place Jussieu, F-75230 Paris Cedex 05, France

Commun. Math. Phys. 97, 465-471 (1985)

Due to an unfortunate error, the following note was omitted.

Note added in proof. After completion of this work, in collaboration with J. Fröhlich and T. Spencer, we were able to prove the stronger result: $\sigma(H(v))$ $=\sigma_{p p}(H(v))$ a.s. for large disorder, and the exponential localization of the eigenfunctions.

The permanent address of $\mathrm{F}$. Martinelli has changed to: Dipartimento di Matematica, Università "La Sapienza", Piazzale Aldo Moro 2, I-00185 Roma, Italy 